\title{
On a degenerate parabolic problem in Hölder spaces
}

\section{Tarik Berroug a, Hua Ding ${ }^{\text {b }}$, Rabah Labbas ${ }^{\mathrm{c}, *}$, Boubaker-Khaled Sadallah ${ }^{\mathrm{d}}$}

a Université du Havre, LMAH, BP 540, 25 rue Philippe Lebon, 76058 Le Havre Cedex, France

b Division of Engineering Sciences, Institute of Mechanics, CAS, Beijing, China

c Mathematical Laboratory, Université du Havre, LMAH, F.S.T., BP 540, 25 rue Philippe Lebon, F-76058 Le Havre Cedex, France

${ }^{\mathrm{d}}$ Lab. E.D.P. \& Hist. of Maths, Department of Mathematics, Ecole Normale Supérieure, 16050-Kouba, Algiers, Algeria

\begin{abstract}
In this paper, we study some degenerate parabolic equation with Cauchy-Dirichlet boundary conditions. This problem is considered in little Hölder spaces. The optimal regularity of the solution $v$ is obtained and is specified in terms of those of the second member when some conditions upon the Hölder exponent with respect to the degeneracy are satisfied. The proofs mainly use the sum theory of linear operators with or without density of domains and the results of smoothness obtained in the study of some abstract linear differential equations of elliptic type.
\end{abstract}

(C) 2004 Elsevier Inc. All rights reserved.

\section{Introduction}

This paper is devoted to the study of a degenerate parabolic problem in the square $\Omega=] 0,1[\times] 0,1[$ described by the variables $(t, y)$

\footnotetext{
${ }^{*}$ Corresponding author.

E-mail address: labbas@univ-lehavre.fr (R. Labbas).
} 


$$
\left\{\begin{array}{l}
t^{2 \alpha} D_{t} v(t, y)-D_{y}^{2} v(t, y)=k(t, y), \\
v_{\mid \partial \Omega-\Gamma_{1}}=0,
\end{array}\right.
$$

where $\left.\Gamma_{1}=\{1\} \times\right] 0,1[, \alpha \in] 1 / 2, \infty[$ and $k$ is a function belonging to the following weighted subspace

$$
h_{0, \Phi}^{\sigma}(\bar{\Omega})=\left\{k \in h_{0}^{\sigma}(\bar{\Omega}): \Phi(t) k \in h_{0}^{\sigma}(\bar{\Omega})\right\}
$$

of the little Hölder space $h_{0}^{\sigma}(\bar{\Omega}), 0<\sigma<1$, defined in Section 2. The weight $\Phi$ is defined by

$$
\Phi(t)=t^{-2 \alpha} \mathrm{e}^{t^{1-2 \alpha}} .
$$

In this work, we give an alternative approach with respect to the results due to Favini and Yagi [3]. These authors have studied some abstract problems of parabolic type with a degenerate term in the time derivative. They have particularly used the notion of multivalued linear operators and construct fundamental solutions when the right-hand side has a Hölder regularity with respect to the time.

Our techniques are different and use the sum theory of linear operators in two cases:

1. When the domains of these operators are dense.

2. When the operators are not necessarily densely defined.

On the other hand, in order to have some maximal regularities, we will use the optimal results obtained in the study of some abstract linear differential equations of elliptic type by Labbas [4] and El Haial-Labbas [5].

Problem (1) can be regarded as the principal part of the following diffusion problem

$$
\left\{\begin{array}{l}
D_{t} u(t, x)-D_{x}^{2} u(t, x)=f(t, x), \\
u_{\mid \partial T-\Gamma_{1}}=0,
\end{array}\right.
$$

set in the curvilinear triangle $T$

$$
T=\left\{(t, x) \in \mathbb{R}^{2}: t \in\right] 0,1\left[, 0<x<t^{\alpha}=\varphi(t)\right\},
$$

when we make the change of variables and function

$$
\left\{\begin{array}{l}
(t, y)=\left(t, x / t^{\alpha}\right) \\
u(t, x)=v(t, y) .
\end{array}\right.
$$

$T$ is then transformed into $\Omega$.

This last problem represents Fick's second law which modelizes, for instance, the concentration (of atoms) $u(t, x)$ at time $t$ in a position $x$, (like the carburization of steel) in a homogeneous system (pure metal or any alloy). It is 
also the modelization of the lateral diffusion of a polluant in a flow of river with variable width.

We also mention that in the framework of the $L^{p}(T)$-space, with $1<p<\infty$, a study is given in Labbas-Medeghri-Sadallah [7] for Problem (2) where it is proved that the solution $u$ has the optimal smoothness, that is

$$
u \in H_{p}^{1,2}(T)=\left\{\psi \in L^{p}(T): D_{t} \psi, D_{x}^{j} \psi \in L^{p}(T), j=1,2\right\},
$$

provided that $\alpha \in] 1 / 2, p-1\left[\right.$ and $f$ is in some $L^{p}(T)$-weighted subspace.

In this paper our main results (which are the equivalent to those obtained in the $L^{p}(T)$-study mentioned above) are the following:

Theorem 1. For $k$ belonging to the weighted space $h_{0, \Phi}^{\sigma}(\bar{\Omega})$ with $\alpha>(1+\sigma) / 2$, Problem (1) admits a unique strict solution $v \in h_{0}^{\sigma}(\bar{\Omega})$ such that

$$
\left\{\begin{array}{l}
D_{y}^{2} v \in h_{0}^{\sigma}(\bar{\Omega}) \\
t^{2 \alpha} D_{t} v \in h_{0}^{\sigma}(\bar{\Omega})
\end{array}\right.
$$

Moreover $v$ satisfies the vector-valued weight regularities

$$
\left\{\begin{array}{l}
t^{-2 \alpha} v \in h_{0}^{\sigma}(\bar{\Omega}) \\
t^{-2 \alpha} v \in h_{0}^{\sigma}\left([0,1] ; W_{0}^{1, q}(0,1)\right)
\end{array}\right.
$$

for all $q>1$.

\section{On little Hölder spaces}

\subsection{Preliminary results}

In this section $\left(X,\|\cdot\|_{X}\right)$ stands for a complex Banach space, $Q$ is an open set of $\mathbb{R}^{n}$ (not necessarily bounded) and $l \in \mathbb{N}$. We denote by $C_{b}^{l}(Q ; X)$ the space of the vector-valued functions with continuous and bounded derivatives up to the order $l$ in $Q$. In the case $X=\mathbb{C}$, one will simply write $C_{b}^{l}(Q)$ instead of $C_{b}^{l}(Q ; \mathbb{C})$.

For $\sigma \in] 0,1\left[\right.$, the Banach space $C^{\sigma}(Q ; X)$ denotes the space of the bounded and $\sigma$-Hölder continuous functions $f: Q \rightarrow X$, such that

$$
\left\{\begin{array}{l}
\sup _{x \in Q}\|f(x)\|_{X}<\infty \\
\exists C>0: \forall x, y \in Q, \quad\|f(x)-f(y)\|_{X} \leqslant C\|x-y\|^{\sigma},
\end{array}\right.
$$

endowed with the norm

$$
\|f\|_{C^{\sigma}(Q ; X)}=\sup _{x \in Q}\|f(x)\|_{X}+\sup _{x \neq y} \frac{\|f(x)-f(y)\|_{X}}{\|x-y\|^{\sigma}}=\|f\|_{\infty}+[f]_{C^{\sigma}(Q ; X)} .
$$

For simplicity, we shall write $C^{\sigma}(Q)$ instead of $C^{\sigma}(Q ; \mathbb{C})$. 
It is well known that any function $f \in C^{\sigma}(Q ; X)$, with $\left.\sigma \in\right] 0,1[$, can be extended to a function of $C^{\sigma}(\bar{Q} ; X)$. This is why we shall write in the sequel $C^{\sigma}(Q ; X)$ or $C^{\sigma}(\bar{Q} ; X)$, or briefly $C^{\sigma}(X)$.

The little Hölder Banach space $h^{\sigma}(Q ; X)$ is defined by

$$
h^{\sigma}(Q ; X)=\left\{f \in C^{\sigma}(Q ; X): \lim _{\delta \rightarrow 0} \sup _{0<\|x-y\| \leqslant \delta} \frac{\|f(x)-f(y)\|_{X}}{\|x-y\|^{\sigma}}=0\right\},
$$

the norm $\|\cdot\|_{h^{\sigma}(Q ; X)}$ is that inducted by $\|\cdot\|_{C^{\sigma}(Q ; X)}$. We can show that

1. for any $\epsilon \in] 0, \sigma\left[\right.$ and $l \in \mathbb{N}^{*} \backslash\{1\}$, we have

$$
\begin{aligned}
C_{b}^{l}(Q ; X) & \subset C_{b}^{1}(Q ; X) \subset h^{\sigma}(Q ; X) \subset C^{\sigma}(Q ; X) \subset h^{\sigma-\epsilon}(Q ; X) \subset C^{\sigma-\epsilon}(Q ; X) \\
& \subset C_{b}^{0}(Q ; X),
\end{aligned}
$$

2. $f \in h^{\sigma}(Q ; X)$ if and only if

$$
\left\{\begin{array}{l}
\forall \delta>0, \exists C(\delta)>0: \forall x, y \in Q, \\
\|x-y\| \leqslant \delta \Rightarrow\|f(x)-f(y)\|_{X} \leqslant C(\delta)\|x-y\|^{\sigma} \\
\text { with } \lim _{\delta \rightarrow 0^{+}} C(\delta)=0,
\end{array}\right.
$$

3. for all $\sigma \in] 0,1\left[\right.$, every function of $h^{\sigma}(Q ; X)$ can be extended to a function of $h^{\sigma}(\bar{Q} ; X)$.

One of the main results concerning the little Hölder spaces is the following density property (see Lunardi [8]).

Proposition 2. Let $Q$ be an open set with regular boundary of $\mathbb{R}^{n}$ or a (possibly unbounded) interval of $\mathbb{R}$. For every $\sigma \in] 0,1[$ and $l \in] \sigma,+\infty\left[\right.$, the space $h^{\sigma}\left(\mathbb{R}^{n} ; X\right)$ (resp. $\left.h^{\sigma}(\bar{Q} ; X)\right)$ is the closure of $C^{l}\left(\mathbb{R}^{n} ; X\right)\left(\right.$ resp. $\left.C^{l}(\bar{Q} ; X)\right)$ in $C^{\sigma}\left(\mathbb{R}^{n} ; X\right)($ resp. $\left.C^{\sigma}(\bar{Q} ; X)\right)$.

We have obtained a similar result in the curvilinear triangle $T$

$$
T=\left\{(t, x) \in \mathbb{R}^{2}: t \in\right] 0,1\left[\text { and } 0<x<t^{\alpha}=\varphi(t)\right\}
$$

(see [1]).

We will use the notations

$$
\begin{aligned}
& h_{0}^{\sigma}(\bar{\Omega})=\left\{v \in h^{\sigma}(\bar{\Omega}): v_{\mid \rho \Omega-\Gamma_{1}}=0\right\}, \\
& h_{0,0}^{\sigma}([0,1])=\left\{\phi \in h^{\sigma}([0,1]): \phi(0)=\phi(1)=0\right\}, \\
& h_{0}^{\sigma}([0,1] ; X)=\left\{N \in h^{\sigma}([0,1] ; X): N(0)=0\right\}
\end{aligned}
$$


and

$$
\begin{aligned}
& C_{0}([0,1])=\{\phi \in C([0,1]): \phi(0)=0\}, \\
& C_{0,0}([0,1])=\{\phi \in C([0,1]): \phi(0)=\phi(1)=0\}, \\
& C_{0,0}^{\sigma}([0,1] ; X)=\left\{N \in C^{\sigma}([0,1] ; X): N(0)=N(1)=0\right\} .
\end{aligned}
$$

The domains of closed linear operators defined in Lebesgue spaces are dense in general. It is not the same in the framework of the spaces of Hölder continuous functions. For example, in the Banach space $C_{0,0}^{\sigma}([0,1] ; X)$ and for the operator $C$ defined by

$$
\left\{\begin{array}{l}
D(C)=\left\{N \in C^{2}([0,1] ; X): N^{\prime \prime} \in C^{\sigma}([0,1] ; X) \text { and } N(0)=N(1)=0\right\}, \\
C(N)=N^{\prime \prime}
\end{array}\right.
$$

the closure of $D(C)$ in $C_{0,0}^{\sigma}([0,1] ; X)$ is exactly

$$
h_{0,0}^{\sigma}([0,1] ; X)=\left\{N \in h^{\sigma}([0,1] ; X): N(0)=N(1)=0\right\} .
$$

The little Hölder spaces are therefore the good appropriated spaces if we want to apply the sum theory of densely defined operators in the Hölder space.

\subsection{Anisotropic properties}

One will need the following lemmas.

Lemma 3. Let $h \in h_{0}^{\sigma}(\bar{\Omega})$. Then the vector-valued function $N$ defined by

$$
N(t)(y)=h(t, y), \quad t \in[0,1]
$$

is in the space

$$
L^{\infty}\left((0,1) ; h_{0,0}^{\sigma}([0,1])\right) \cap h_{0}^{\sigma}\left([0,1] ; C_{0,0}([0,1])\right) .
$$

$L^{\infty}\left((0,1) ; h_{0,0}^{\sigma}([0,1])\right)$ denotes the space of the vector-valued functions $N$ strongly measurable such that $N(t) \in h_{0,0}^{\sigma}([0,1])$ for a.e. $t \in(0,1)$.

Proof. Let $h \in h_{0}^{\sigma}(\bar{\Omega})$.

1. The strong measurability of $N$ is obtained by an analogous argument as in Najmi [9] (p. 95).

2. $\forall \delta>0, \exists C(\delta)$ such that for $\left|y-y^{\prime}\right| \leqslant \delta$ and $t \in[0,1]$, one has

$$
\begin{aligned}
\mid N(t)(y)-N(t)\left(y^{\prime}\right) \| & =\left|h(t, y)-h\left(t, y^{\prime}\right)\right| \leqslant C(\delta)\left\|(t, y)-\left(t, y^{\prime}\right)\right\|^{\sigma} \\
& \leqslant C(\delta)\left|y-y^{\prime}\right|^{\sigma}
\end{aligned}
$$

with $\lim _{\delta \rightarrow 0^{+}} C(\delta)=0$. So $N(t) \in h_{0,0}^{\sigma}([0,1])$. Now for a.e. $t \in(0,1)$ we get

$$
\|N(t)\|_{h^{\sigma}([0,1])}=\max _{y \in[0,1]}|h(t, y)|+\sup _{0<\left|y-y^{\prime}\right| \leqslant \delta} \frac{\left|h(t, y)-h\left(t, y^{\prime}\right)\right|}{\left|y-y^{\prime}\right|^{\sigma}} \leqslant\|h\|_{h_{0}^{\sigma}(\bar{\Omega})} .
$$


Thus $N \in L^{\infty}\left((0,1) ; h_{0,0}^{\sigma}([0,1])\right)$ and

$$
\|N\|_{L^{\infty}\left((0,1) ; h_{0,0}^{\sigma}([0,1])\right)} \leqslant\|h\|_{h_{0}^{\sigma}(\bar{\Omega})} .
$$

3. $\forall \delta>0, \exists C_{1}(\delta)$ such that for $\|t-\tau\| \leqslant \delta$ one has

$$
\|N(t)-N(\tau)\|_{C_{0,0}([0,1])}=\max _{y \in[0,1]}|h(t, y)-h(\tau, y)| \leqslant C_{1}(\delta)|t-\tau|^{\sigma}
$$

with $\lim _{\delta \rightarrow 0^{+}} C_{1}(\delta)=0$. On the other hand

$$
N(0)(y)=h(0, y)=0 .
$$

Then $N \in h_{0}^{\sigma}\left([0,1] ; C_{0,0}([0,1])\right)$.

It is not difficult to deduce the following lemma which specifies the relation between global and anisotropic regularities in the above spaces.

Lemma 4. One has

$$
h_{0}^{\sigma}(\bar{\Omega})=L^{\infty}\left((0,1) ; h_{0,0}^{\sigma}([0,1])\right) \cap h_{0}^{\sigma}\left([0,1] ; C_{0,0}([0,1])\right) .
$$

\section{Sums of linear operators}

Let us now recall the essential of the sum theory we will have to apply.

Let $A$ and $B$ be two closed linear operators in a complex Banach $E$, with domains $D(A)$ and $D(B)$. We assume that there exist positive constants $C_{A}, C_{B}$, $\epsilon_{A}, \epsilon_{B}$ such that

(D.G.0) $\overline{D(A)+D(B)}=E$,

$$
\begin{gathered}
\text { (D.G.1) }\left\{\begin{array}{l}
\left(\text { i) } \rho(-A) \supset \sum_{\epsilon_{A}}=\left\{z \in \mathbb{C}^{*}:|\arg (z)|<\epsilon_{A}\right\}\right. \\
\forall z \in \sum_{\epsilon_{A}}\left\|(A+z I)^{-1}\right\|_{L(E)} \leqslant C_{A} /|z|, \\
\left(\text { ii) } \rho(-B) \supset \sum_{\epsilon_{B}}=\left\{z \in \mathbb{C}^{*}:|\arg (z)|<\epsilon_{B}\right\}\right. \\
\forall z \in \sum_{\epsilon_{B}}\left\|(B+z I)^{-1}\right\|_{L(E)} \leqslant C_{B} /|z|, \\
\left(\text { iii) } \epsilon_{A}+\epsilon_{B}>\pi,\right. \\
(\text { iv) } \sigma(-A) \cap \sigma(B)=\emptyset,
\end{array}\right. \\
\text { (D.G.2) }\left\{\begin{array}{l}
\forall \xi \in \rho(-A), \forall \eta \in \rho(-B), \\
(A+\xi I)^{-1}(B+\eta I)^{-1}=(B+\eta I)^{-1}(A+\xi I)^{-1},
\end{array}\right.
\end{gathered}
$$

where $\rho(-A)$ and $\rho(-B)$ are the resolvent sets of $-A$ and $-B, \sigma(-A)$ and $\sigma(B)$ are the spectra of $-A$ and $B$. 
Due to Da Prato-Grisvard [2], we have the two following results.

Theorem 5. Assume (D.G.0), (D.G.1) and (D.G.2). Then for any $\lambda>0$, the sum

$$
A+B+\lambda I
$$

is closable and $0 \in \rho(\overline{A+B+\lambda I})$.

The inverse of $\overline{A+B+\lambda I}$ is given by the Dunford's integral

$$
L_{\lambda}=\frac{-1}{2 \mathrm{i} \pi} \int_{\Gamma}(B-z I)^{-1}(A+z I+\lambda I)^{-1} \mathrm{~d} z,
$$

where $\Gamma$ is a sectorial curve separating $\sigma(-A)$ and $\sigma(B)$ and lying in $\rho(-A) \cap \rho(B)$. The function

$$
w=(\overline{A+B+\lambda I})^{-1}(f)=L_{\lambda}(f)
$$

is called a strong solution of equation $A w+B w+\lambda w=f$.

Theorem 6. Assume (D.G.0), (D.G.1) and (D.G.2). Let F be a Banach subspace containing $D(A)$ and continuously imbedded in $E$ such that there exists a constant $K$ satisfying, for some $\theta \in] 0,1[$,

$$
\forall x \in D(A), \quad\|x\|_{F} \leqslant K\left(\|x\|_{E}+\|x\|_{E}^{1-\theta}\|A x\|_{E}^{\theta}\right) .
$$

Then $D(\overline{A+B}) \subset F$.

When neither $D(A)$, nor $D(B)$ is dense in $E$, Labbas [6] has proved the following result.

Theorem 7. Assume (D.G.1) and (D.G.2). Then for any $\lambda>0$, there exists a closed extension $\widetilde{A+B}$ such that

$$
L_{\lambda}=(\widetilde{A+B}+\lambda I)^{-1} \text {. }
$$

In this case $A+B$ is closable and thus $\overline{D(A+B)} \subset D(\widetilde{A+B})$. But in [6], we can find an example where $\overline{D(A+B)}$ is strictly imbedded in $D(\overline{A+B})$.

\section{Proof of Theorem 1}

4.1. Writing Problem (1) in an operational form

Let us go back to our problem we started from

$$
\left\{\begin{array}{l}
t^{2 \alpha} D_{t} v(t, y)-D_{y}^{2} v(t, y)=k(t, y), \\
v_{\mid \partial \Omega-\Gamma_{1}}=0
\end{array}\right.
$$


where $k \in h_{0, \Phi}^{\sigma}(\bar{\Omega})$ and

$$
\alpha>\frac{1+\sigma}{2} \text {. }
$$

Set

$$
g(t, y)=t^{-2 \alpha} k(t, y)
$$

then

$$
g \in E_{\exp , \Omega}=\left\{\psi \in h_{0}^{\sigma}(\bar{\Omega}): \mathrm{e}^{t^{1-2 \alpha}} \psi \in h_{0}^{\sigma}(\bar{\Omega})\right\} .
$$

Remark 8. Observe that if $f$ (defined on the curvilinear triangle) is in the space

$$
E_{\exp , T}=\left\{f \in h_{0}^{\sigma}(\bar{T}): \mathrm{e}^{t^{1-2 \alpha}} f \in h_{0}^{\sigma}(\bar{T})\right\},
$$

then the corresponding function $g$ defined on the square $\Omega$ by

$$
g(t, y)=f(t, x), \quad y=x / t^{\alpha}
$$

belongs to $E_{\exp , \Omega}$.

Let us set

$$
\left\{\begin{array}{l}
w(t, y)=\mathrm{e}^{-\lambda \frac{1-2 \alpha}{(1-2 \alpha)}} v(t, y) \\
h(t, y)=\mathrm{e}^{-\lambda \frac{t^{1}-2 \alpha}{(1-2 \alpha)}} k(t, y)
\end{array}\right.
$$

where $\lambda$ is a fixed number in $] 0,2 \alpha-1[$. Then $w$ verifies

$$
t^{2 \alpha} D_{t} w(t, y)-D_{y}^{2} w(t, y)+\lambda w(t, y)=h(t, y)
$$

and

$$
\begin{aligned}
t^{-2 \alpha} h(t, y) & =\mathrm{e}^{-\lambda \frac{t^{1-2 \alpha}}{(1-2 \alpha)}} \mathrm{e}^{-t^{1-2 \alpha}}\left(\mathrm{e}^{t^{1-2 \alpha}} t^{-2 \alpha} k(t, y)\right)=\mathrm{e}^{\left(\frac{\lambda}{2 \alpha-1}-1\right) t^{1-2 \alpha}}\left(\mathrm{e}^{t^{1-2 \alpha}} t^{-2 \alpha} k(t, y)\right) \\
& =\mathrm{e}^{\left(\frac{\lambda}{2 \alpha-1}-1\right) t^{1-2 \alpha}}(\Phi(t) k(t, y))
\end{aligned}
$$

Hence, thanks to the fact that the function $x$ defined by

$$
x(t)=\mathrm{e}^{\left(\frac{\lambda}{2 \alpha-1}-1\right) t^{1-2 \alpha}}
$$

is in $C_{b}^{1}([0,1])$ (since $\left.\lambda \in\right] 0,2 \alpha-1[$ ), we deduce that

$$
t^{-2 \alpha} h \in h_{0}^{\sigma}(\bar{\Omega}) \text {. }
$$

Thus, for the resolution of Problem (1), it is sufficient to solve

$$
\left\{\begin{array}{l}
t^{2 \alpha} D_{t} w(t, y)-D_{y}^{2} w(t, y)+\lambda w(t, y)=h(t, y), \\
w_{\mid \partial \Omega-\Gamma_{1}}=0
\end{array}\right.
$$


in the weighted Banach space

$$
E=\left\{h \in h_{0}^{\sigma}(\bar{\Omega}): t^{-2 \alpha} h \in h_{0}^{\sigma}(\bar{\Omega})\right\},
$$

equipped with the norm

$$
\|h\|_{E}=\left\|t^{-2 \alpha} h\right\|_{h^{\sigma}(\bar{\Omega})}+\|h\|_{h^{\sigma}(\bar{\Omega})} .
$$

Now, let us define the following vector-valued functions

$$
\begin{aligned}
& N:[0,1] \rightarrow X ; t \rightarrow N(t) ; N(t)(y)=h(t, y), \\
& M:[0,1] \rightarrow X ; t \rightarrow M(t) ; M(t)(y)=w(t, y),
\end{aligned}
$$

Then we obtain the operational form of the previous problem, mainly

$$
\left\{\begin{array}{l}
t^{2 \alpha} D_{t} M(t)+L M(t)+\lambda M(t)=N(t), \\
M(0)=0,
\end{array}\right.
$$

where $L$ is a linear operator with domain $D(L)$ in some Banach space $X$ which will be specified later on.

Thanks to lemmas 3 and 4, the second member $N$ is such that

$$
\left\{\begin{array}{l}
N \in L^{\infty}\left((0,1) ; h_{0,0}^{\sigma}([0,1])\right) \cap h_{0}^{\sigma}\left([0,1] ; C_{0,0}([0,1])\right), \\
t^{-2 \alpha} N \in L^{\infty}\left((0,1) ; h_{0,0}^{\sigma}([0,1])\right) \cap h_{0}^{\sigma}\left([0,1] ; C_{0,0}([0,1])\right) .
\end{array}\right.
$$

\subsection{First application of the sums}

In this subsection we choose $X=h_{0,0}^{\sigma}([0,1])$. Our goal is to apply the results of Section 3 to Problem (7) in the Banach space

$$
E_{\infty}=\left\{N \in L^{\infty}((0,1) ; X): t^{-2 \alpha} N \in L^{\infty}((0,1) ; X)\right\}
$$

equipped with the norm

$$
\|N\|_{E_{\infty}}=\sup _{t \in(0,1)}\|N(t)\|_{X}+\sup _{t \in(0,1)}\left\|t^{-2 \alpha} N(t)\right\|_{X} .
$$

Let us define the following three operators $L, A$ and $B$ by

$$
\begin{aligned}
& \left\{\begin{array}{l}
D(L)=\left\{\phi \in h_{0,0}^{\sigma}([0,1]): \phi^{\prime}, \phi^{\prime \prime} \in h_{0,0}^{\sigma}([0,1])\right\}, \\
(L \phi)(y)=-\phi^{\prime \prime}(y),
\end{array}\right. \\
& \left\{\begin{array}{l}
D(A)=\left\{M \in E_{\infty}: M(t) \in D(L), \text { a.e. } t \in(0,1)\right\}, \\
(A M)(t)=L(M(t)), \quad t \in(0,1)
\end{array}\right.
\end{aligned}
$$

and

$$
\left\{\begin{array}{l}
D(B)=\left\{M \in E_{\infty}: t^{2 \alpha} D_{t} M \in E_{\infty}, M(0)=0\right\} \\
(B M)(t)=t^{2 \alpha} D_{t} M(t), \quad t \in(0,1)
\end{array}\right.
$$


keeping in mind that the derivative $D_{t} M$ understood in the context of derivation in the vector-valued distributions space

$$
D^{\prime}(] 0,1\left[; h_{0,0}^{\sigma}([0,1])\right),
$$

since

$$
E_{\infty} \subset L^{\infty}\left((0,1) ; h_{0,0}^{\sigma}([0,1])\right) \subset D^{\prime}(] 0,1\left[; h_{0,0}^{\sigma}([0,1])\right) .
$$

Problem (7) is then equivalent to

$$
A M+B M+\lambda M=N .
$$

The spectral properties of $A$ and $B$ are as follows.

Proposition 9. $A$ and B satisfy Assumptions (D.G.1) and (D.G.2).

\section{Proof}

1. First, observe that the domains $D(A)$ and $D(B)$ are not dense in $E_{\infty}$. The operator $A$ has the same properties as its realization $L$. By direct computations, we prove, for each $\left.\epsilon_{0} \in\right] 0, \pi[$, that

$$
\rho(-L) \supset \Sigma_{\pi-\epsilon_{0}}=\left\{z \in \mathbb{C}^{*}:\left\{|\arg (z)|<\pi-\epsilon_{0}\right\}\right.
$$

and

$$
\forall z \in \Sigma_{\pi-\epsilon_{0}}\left\|(-L+\lambda)^{-1}\right\|_{L(X)} \leqslant \frac{1}{|z| \cos \left(\frac{\pi-\epsilon_{0}}{2}\right)} .
$$

Then

$$
\rho(-A) \supset \Sigma_{\pi-\epsilon_{0}}
$$

and

$$
\forall z \in \Sigma_{\pi-\epsilon_{0}}\left\|(A+z)^{-1}\right\|_{L\left(E_{\infty}\right)} \leqslant \frac{1}{|z| \cos \left(\frac{\pi-\epsilon_{0}}{2}\right)} .
$$

Therefore $A$ verifies (D.G.1) statement (i) with $\epsilon_{A}=\pi-\epsilon_{0}$.

2. Now, concerning the operator $B$, we first verify that the domain $D(B)$ is well defined. Indeed, if $M \in D(B)$ one has

$$
\left\{\begin{array}{l}
M \in L^{\infty}((0,1) ; X), \\
t^{-2 \alpha} M \in L^{\infty}((0,1) ; X), \\
t^{2 \alpha} D_{t} M \in L^{\infty}((0,1) ; X), \\
t^{-2 \alpha}\left(t^{2 \alpha} D_{t} M\right)=D_{t} M \in L^{\infty}((0,1) ; X) .
\end{array}\right.
$$

Moreover it is well known that if $M$ and $D_{t} M$ belong to $L^{\infty}((0,1) ; X)$ then $M$ admits a continuous representation on $[0,1]$, thus $M(0)=0$ has a sense. 
3. Let us study the spectral equation

$$
B M+z M=h,
$$

where $h \in E_{\infty}$.

If $\operatorname{Re}(z)>0$, the solution of the problem

$$
\left\{\begin{array}{l}
t^{2 \alpha} M^{\prime}(t)+z M(t)=h(t), \\
M(0)=0
\end{array}\right.
$$

is given by

$$
M(t)=\int_{0}^{t}\left(\frac{h(\tau)}{\tau^{2 \alpha}} \mathrm{e}^{-z \int_{\tau}^{t} s^{-2 \alpha} \mathrm{d} s}\right) \mathrm{d} \tau .
$$

On the other hand, one has, for a.e. $t \in(0,1)$

$$
\begin{aligned}
\|M(t)\|_{X} & \leqslant \int_{0}^{t}\left(\|h(\tau)\|_{X} \tau^{-2 \alpha} \mathrm{e}^{-\operatorname{Re}(z) \int_{\tau}^{t} s^{-2 \alpha} \mathrm{d} s}\right) \mathrm{d} \tau \\
& \leqslant\|h\|_{E_{\infty}} \int_{0}^{t} \tau^{-2 \alpha} \mathrm{e}^{-\operatorname{Re}(z) \int_{\tau}^{t} s^{-2 \alpha} \mathrm{d} s} \mathrm{~d} \tau \\
& \leqslant\|h\|_{E_{\infty}} \int_{0}^{t} \tau^{-2 \alpha} \mathrm{e}^{\frac{\operatorname{Re}(z)}{2 \alpha-1}}\left(t^{1-2 \alpha-\tau^{1-2 \alpha}}\right) \mathrm{d} \tau \\
& \leqslant\|h\|_{E_{\infty}} \mathrm{e}^{\frac{\operatorname{Re}(z)}{2 \alpha-1} t^{-2 \alpha}} \int_{0}^{t} \tau^{-2 \alpha} \mathrm{e}^{\frac{-\operatorname{Re}(z)}{2 \alpha-1} \tau^{1-2 \alpha}} \mathrm{d} \tau \leqslant \frac{\|h\|_{E_{\infty}}}{\operatorname{Re}(z)}
\end{aligned}
$$

and

$$
\begin{aligned}
\left\|t^{-2 \alpha} M(t)\right\|_{X} & \leqslant \int_{0}^{t}\left(t^{-2 \alpha}\|h(\tau)\|_{X} \tau^{-2 \alpha} \mathrm{e}^{-\operatorname{Re} z \int_{\tau}^{t} s^{-2 \alpha} \mathrm{d} s}\right) \mathrm{d} \tau \\
& =\int_{0}^{t}\left(t^{-2 \alpha} \tau^{2 \alpha}\left\|\tau^{-2 \alpha} h(\tau)\right\|_{X} \tau^{-2 \alpha} \mathrm{e}^{-\operatorname{Re} z \int_{\tau}^{t} s^{-2 \alpha} \mathrm{d} s}\right) \mathrm{d} \tau \\
& \leqslant\|h\|_{E_{\infty}} \int_{0}^{t} \tau^{-2 \alpha} \mathrm{e}^{-\operatorname{Re} z \int_{\tau}^{t} s^{-2 \alpha} \mathrm{d} s} \mathrm{~d} \tau \leqslant \frac{\|h\|_{E_{\infty}}}{\operatorname{Re}(z)}
\end{aligned}
$$

Then $M \in E_{\infty}, M(0)=0$ and

$$
\left\|(B+z)^{-1}\right\|_{L\left(E_{\infty}\right)} \leqslant \frac{1}{\operatorname{Re}(z)} .
$$

Hence, for all $\left.\epsilon_{1} \in\right] 0, \pi / 2[$

$$
\rho(-B) \supset \Sigma_{\pi / 2-\epsilon_{1}}=\left\{z \in \mathbb{C}^{*}: \mid \arg (z) \|<\pi / 2-\epsilon_{1}=\epsilon_{B}\right\} .
$$

Now, let us choose $\epsilon_{1}$ such that $\pi / 2-\epsilon_{1}>\epsilon_{0}$, then

$$
\epsilon_{A}+\epsilon_{B}=\left(\pi-\epsilon_{0}\right)+\left(\pi / 2-\epsilon_{1}\right)>\pi .
$$

Summing up we have (D.G.1). 
The assumption of commutativity (D.G.2) is checked by direct computations.

Observe that, thanks to Theorem $7, A+B$ admits a closed extension $\widetilde{A+B}$ and, for all $\lambda>0$

$$
(\widetilde{A+B}+\lambda I)^{-1}=L_{\lambda}
$$

which means that, for $N \in E_{\infty}$, there exists a unique $M \in E_{\infty}$ such that

$$
\left\{\begin{array}{l}
M \in D(\widetilde{A+B}) \\
(\widetilde{A+B}+\lambda I) M=N .
\end{array}\right.
$$

We then deduce the following result corresponding to $w_{1}$ defined by $w_{1}(t, y)=M(t)(y)$.

Proposition 10. For $h \in E$, there exists a unique $w_{1} \in E_{\infty}$ solution in the sense of $D^{\prime}(] 0,1\left[; h_{0,0}^{\sigma}([0,1])\right)$ of the equation

$$
t^{2 \alpha} D_{t} w_{1}(t, .)-D_{y}^{2} w_{1}(t, .)+\lambda w_{1}(t, .)=h(t, .)
$$

\subsection{Second application of the sums}

Now, set $X=C_{0,0}([0,1])$ and consider in the Banach space

$$
E_{\mathrm{hol}}=\left\{N \in h_{0}^{\sigma}([0,1] ; X): t^{-2 \alpha} N \in h_{0}^{\sigma}([0,1] ; X)\right\},
$$

the operators $L, A$ and $B$ defined by

$$
\begin{aligned}
& \left\{\begin{array}{l}
D(L)=\left\{\phi \in C_{0,0}([0,1]) \cap W^{2, q}(0,1), q>1: \phi^{\prime}, \phi^{\prime \prime} \in C_{0,0}([0,1])\right\}, \\
(L \phi)(y)=-\phi^{\prime \prime}(y),
\end{array}\right. \\
& \left\{\begin{array}{l}
D(A)=\left\{M \in E_{\mathrm{hol}}: \forall t \in[0,1] M(t) \in D(L)\right\}, \\
(A M)(t)=L(M(t)), \quad t \in[0,1]
\end{array}\right.
\end{aligned}
$$

and

$$
\left\{\begin{array}{l}
D(B)=\left\{M \in E_{\mathrm{hol}}: \forall t \in(0,1) t^{2 \alpha} D_{t} M \in E_{\mathrm{hol}}\right\} \\
(B M)(t)=t^{2 \alpha} D_{t} M(t), \quad t \in(0,1)
\end{array}\right.
$$

We have

Proposition 11. $A$ and B satisfy (D.G.0), (D.G.1) and (D.G.2) in $E_{\mathrm{hol}}$. 
Proof. It is not difficult to prove that for each $\left.\epsilon_{0} \in\right] 0, \pi[$,

$$
\rho(-A) \supset \Sigma_{\pi-\epsilon_{0}}
$$

and

$$
\forall z \in \Sigma_{\pi-\epsilon_{0}}\left\|(A+z)^{-1}\right\|_{L\left(E_{\mathrm{hol}}\right)} \leqslant \frac{1}{|z| \cos \left(\frac{\pi-\epsilon_{0}}{2}\right)} .
$$

If $M \in D(B)$, then

$$
\left\{\begin{array}{l}
M \in h_{0}^{\sigma}([0,1] ; X) \\
t^{-2 \alpha} M \in h_{0}^{\sigma}([0,1] ; X), \\
t^{2 \alpha} D_{t} M \in h_{0}^{\sigma}([0,1] ; X) \\
t^{-2 \alpha}\left(t^{2 \alpha} D_{t} M\right)=D_{t} M \in h_{0}^{\sigma}([0,1] ; X) .
\end{array}\right.
$$

Therefore the operator $B$ is clearly well defined. It is enough to check the spectral properties and the density. For the latter, one will use the density of the space $C^{2}([0,1] ; X) \cap C_{0}([0,1] ; X)$ in $h_{0}^{\sigma}([0,1] ; X)$ assuming that $h_{0}^{\sigma}([0,1] ; X)$ is equipped with the norm of the Hölder space $C^{\sigma}([0,1] ; X)$.

Let us show that the space

$$
\mathscr{E}_{t^{2 \alpha}}=\left\{t^{2 \alpha} \psi: \psi \in C^{2}([0,1] ; X) \cap C_{0}([0,1] ; X)\right\}
$$

is dense in $E_{\mathrm{hol}}$. For this purpose we consider $N \in E_{\mathrm{hol}}$. Thus

$$
N \in h_{0}^{\sigma}([0,1] ; X) \quad \text { and } \quad t^{-2 \alpha} N \in h_{0}^{\sigma}([0,1] ; X) .
$$

Putting $t^{-2 \alpha} N=G$, there exists a sequence

$$
G_{n} \in C^{2}([0,1] ; X) \cap C_{0}([0,1] ; X)
$$

such that

$$
\left\|G-G_{n}\right\|_{h^{\sigma}(X)} \rightarrow 0 \quad \text { as } n \rightarrow \infty .
$$

Now, let us define the sequence $N_{n}$ by $N_{n}=t^{2 \alpha} G_{n}$. Then, for $n \rightarrow \infty$

$$
\left\|N-N_{n}\right\|_{h^{\sigma}([0,1] ; X)}=\left\|t^{2 \alpha} G-t^{2 \alpha} G_{n}\right\|_{h^{\sigma}([0,1] ; X)} \rightarrow 0,
$$

hence

$$
\begin{aligned}
\left\|N-N_{n}\right\|_{E_{\mathrm{hol}}} & =\left\|N-N_{n}\right\|_{h^{\sigma}([0,1] ; X)}+\left\|t^{-2 \alpha} N-t^{-2 \alpha} N_{n}\right\|_{h^{\sigma}([0,1] ; X)} \\
& =\left\|N-N_{n}\right\|_{h^{\sigma}([0,1] ; X)}+\left\|G-G_{n}\right\|_{h^{\sigma}([0,1] ; X)} \rightarrow 0 .
\end{aligned}
$$

On the other hand, $\mathscr{E}_{t^{2 \alpha}} \subset D(B)$. Indeed if $M \in \mathscr{E}_{t^{2 \alpha}}$, one has

$$
M=t^{2 \alpha} \psi,
$$

where $\psi \in C^{2}([0,1] ; X) \cap C_{0}([0,1] ; X)$. So 


$$
\left\{\begin{array}{l}
t^{2 \alpha} \psi \in h_{0}^{\sigma}([0,1] ; X), \\
t^{-2 \alpha}\left(t^{2 \alpha} \psi\right)=\psi \in h_{0}^{\sigma}([0,1] ; X)
\end{array}\right.
$$

and

$$
\left\{\begin{array}{l}
t^{2 \alpha} D_{t}\left(t^{2 \alpha} \psi\right)=2 \alpha t^{2 \alpha} t^{2 \alpha-1} \psi+t^{2 \alpha} t^{2 \alpha} D_{t} \psi \in h_{0}^{\sigma}([0,1] ; X), \\
t^{-2 \alpha}\left(t^{2 \alpha} D_{t}\left(t^{2 \alpha} \psi\right)\right)=2 \alpha t^{2 \alpha-1} \psi+t^{2 \alpha} D_{t} \psi \in h_{0}^{\sigma}([0,1] ; X),
\end{array}\right.
$$

the function $t \mapsto t^{2 \alpha-1}$ belongs to $h_{0}^{\sigma}([0,1] ; X)$ since $\alpha>(1+\sigma) / 2$. Hence $\mathscr{E}_{t^{2 \alpha}} \subset D(B)$. We deduce the density of $D(B)$ in $E_{\text {hol }}$ and (D.G.0) follows.

Now, for $h \in E_{\text {hol }}$, the spectral equation

$$
\left\{\begin{array}{l}
t^{2 \alpha} M^{\prime}(t)+z M(t)=h(t) \\
M(0)=0
\end{array}\right.
$$

for $\operatorname{Re}(z)>0$, admits a unique solution formally given by

$$
M(t)=\int_{0}^{t}\left(\frac{h(\tau)}{\tau^{2 \alpha}} \mathrm{e}^{-z \int_{\tau}^{t} s^{-2 \alpha} \mathrm{d} s}\right) \mathrm{d} \tau
$$

Moreover, one has, for all $t \in(0,1)$

$$
\begin{aligned}
\|M(t)\|_{X} & \leqslant \int_{0}^{t}\left(\|h(\tau)\|_{X} \tau^{-2 \alpha} \mathrm{e}^{-\operatorname{Re}(z) \int_{\tau}^{t} s^{-2 \alpha} \mathrm{d} s}\right) \mathrm{d} \tau \\
& \leqslant\|h\|_{E_{\mathrm{hol}}} \int_{0}^{t} \tau^{-2 \alpha} \mathrm{e}^{-\operatorname{Re}(z) \int_{\tau}^{t} s^{-2 \alpha} \mathrm{d} s} \mathrm{~d} \tau \\
& \leqslant\|h\|_{E_{\mathrm{hol}}} \int_{0}^{t} \tau^{-2 \alpha} \mathrm{e}^{\frac{\operatorname{Re}(z)}{2 \alpha-1}\left(t^{1-2 \alpha}-\tau^{1-2 \alpha}\right)} \mathrm{d} \tau \\
& \leqslant\|h\|_{E_{\mathrm{hol}}} \mathrm{e}^{\frac{\operatorname{Re}(z)}{2 \alpha-1} t^{1-2 \alpha}} \int_{0}^{t} \tau^{-2 \alpha} \mathrm{e}^{\frac{-\mathrm{Re} z}{2 \alpha-1} \tau^{1-2 \alpha}} \mathrm{d} \tau \\
& \leqslant \frac{\|h\|_{E_{\mathrm{hol}}}}{\operatorname{Re}(z)}
\end{aligned}
$$

and

$$
\begin{aligned}
\left\|t^{-2 \alpha} M(t)\right\|_{X} & \leqslant \int_{0}^{t}\left(t^{-2 \alpha}\|h(\tau)\|_{X} \tau^{-2 \alpha} \mathrm{e}^{-\operatorname{Re}(z) \int_{\tau}^{t} s^{-2 \alpha} \mathrm{d} s}\right) \mathrm{d} \tau \\
& \leqslant \int_{0}^{t}\left(t^{-2 \alpha} \tau^{2 \alpha}\left\|\tau^{-2 \alpha} h(\tau)\right\|_{X} \tau^{-2 \alpha} \mathrm{e}^{-\operatorname{Re} z \int_{\tau}^{t} s^{-2 \alpha} \mathrm{d} s}\right) \mathrm{d} \tau \\
& \leqslant\|h\|_{E_{\mathrm{hol}}} \int_{0}^{t} \tau^{-2 \alpha} \mathrm{e}^{-\operatorname{Re}(z) \int_{\tau}^{t} s^{-2 \alpha} \mathrm{d} s} \mathrm{~d} \tau \\
& \leqslant \frac{\|h\|_{E_{\mathrm{hol}}}}{\operatorname{Re}(z)}
\end{aligned}
$$


We deduce from these estimates that $M \in E_{\mathrm{hol}}, M(0)=0$ and

$$
\left\|(B+z)^{-1}\right\|_{L\left(E_{\mathrm{hol}}\right)} \leqslant \frac{1}{\operatorname{Re}(z)} .
$$

Hence, for all $\left.\epsilon_{1} \in\right] 0, \pi / 2[$

$$
\rho(-B) \supset \Sigma_{\pi / 2-\epsilon_{1}}=\left\{z \in \mathbb{C}^{*},|\operatorname{Arg}(z)|<\pi / 2-\epsilon_{1}=\epsilon_{B}\right\} .
$$

Assumption (D.G.1) is then verified as above. This ends the proof of the proposition.

Using Theorem 5 for all $q>1$, we deduce that the operator $(A+B)$ is closable, $\overline{A+B}+\lambda I$ is invertible and its inverse coincides with the operator $L_{\lambda}$. This leads to

Proposition 12. For $h \in E_{\mathrm{hol}}$ Problem (6) admits a unique strong solution $w_{2}$ such that

$$
\left\{\begin{array}{l}
w_{2} \in h_{0}^{\sigma}([0,1] ; X) \\
t^{-2 \alpha} w_{2} \in h_{0}^{\sigma}([0,1] ; X)
\end{array}\right.
$$

This result implies, that there exists a sequence $\left(\chi_{n}\right) \in D(A) \cap D(B)$ such that

$$
\left\{\begin{array}{l}
\chi_{n} \rightarrow^{E_{\mathrm{hol}}} w_{2}, \\
t^{2 \alpha} D_{t \chi_{n}}(., .)-D_{y}^{2} \chi_{n}(., .)+\lambda \chi_{n}(., .) \rightarrow^{E_{\mathrm{hol}}} h(., .) .
\end{array}\right.
$$

Then $w_{2}$ is a distribution solution (i.e. $\left.w_{2} \in D^{\prime}(] 0,1\left[; C_{0,0}([0,1])\right)\right)$ of the equation

$$
t^{2 \alpha} D_{t} w(t, .)-D_{y}^{2} w(t, .)+\lambda w(t, .)=h(t, .) .
$$

It is also a distribution solution in $D^{\prime}(] 0,1\left[; h_{0,0}^{\sigma}([0,1])\right)$ and consequently coincides with $w_{1}$. We shall note $w=w_{1}=w_{2}$.

In order to prove that the convexity inequality of Theorem 6 is verified with

$$
F=\left\{M \in h_{0}^{\sigma}\left([0,1] ; W_{0}^{1, q}(0,1)\right): t^{-2 \alpha} M \in h_{0}^{\sigma}\left([0,1] ; W_{0}^{1, q}(0,1)\right)\right\},
$$

we need the following lemma.

Lemma 13. There exists a constant $K>0$ (depending only on $q$ ) such that for all function $\psi \in W^{2, q}(0,1) \cap W_{0}^{1, q}(0,1)$, we have

$$
\|\psi\|_{W_{0}^{1, q}} \leqslant K\|\psi\|_{L^{q}}^{1 / 2}\left\|\psi^{\prime \prime}\right\|_{L^{q}}^{1 / 2} .
$$


Proof. Thanks to Poincaré's inequality, we know that the norms $\|\psi\|_{W_{0}^{1, q}}$ and $\left\|\psi^{\prime}\right\|_{L^{q}}$ are equivalent. Then it is enough to prove that

$$
\left\|\psi^{\prime}\right\|_{L^{q}} \leqslant K\|\psi\|_{L^{q}}^{1 / 2}\left\|\psi^{\prime \prime}\right\|_{L^{q}}^{1 / 2}
$$

Fix a positive number $\mu>1$. Using two integration by parts, we get for a.e. $y \in(0,1)$, the identity

$$
\psi^{\prime}(y)=\int_{0}^{1} G(y, x) \psi^{\prime \prime}(x) \mathrm{d} x+\int_{0}^{1} H(y, x) \psi(x) \mathrm{d} x,
$$

where

$$
G(y, x)= \begin{cases}\frac{x^{\mu+1}}{y^{\mu}} & 0 \leqslant x<y \\ -\frac{(1-x)^{\mu+1}}{(1-y)^{\mu}} & y<x \leqslant 1\end{cases}
$$

and

$$
H(y, x)= \begin{cases}-\frac{\mu(\mu+1) x^{\mu-1}}{y^{\mu}} & 0 \leqslant x<y \\ \frac{\mu(\mu+1)(1-x)^{\mu-1}}{(1-y)^{\mu}} & y<x \leqslant 1\end{cases}
$$

Let us set

$$
\left(K_{1} \psi\right)(y)=\int_{0}^{1} G(y, x) \psi^{\prime \prime}(x) \mathrm{d} x,
$$

then

$$
\sup _{y} \int_{0}^{1}|G(y, x)| \mathrm{d} x \leqslant \sup _{y}\left\{\int_{0}^{y} \frac{x^{\mu+1}}{y^{\mu}} \mathrm{d} x+\int_{y}^{1} \frac{(1-x)^{\mu+1}}{(1-y)^{\mu}} \mathrm{d} x\right\} \leqslant \frac{1}{\mu+2}
$$

and

$$
\sup _{x} \int_{0}^{1}|G(y, x)| \mathrm{d} y \leqslant \frac{1}{\mu-1} .
$$

Thanks to Schur interpolation lemma we obtain

$$
\left\|K_{1}\right\|_{L\left(L^{q}, L^{q}\right)} \leqslant \frac{1}{\mu-1} .
$$

By the same way, putting

$$
\left(K_{2} \psi\right)(y)=\int_{0}^{1} H(y, x) \psi(x) \mathrm{d} x,
$$


we get

$$
\left\|K_{2}\right\|_{L\left(L^{q}, L^{q}\right)} \leqslant \frac{2 \mu(\mu+1)}{\mu-1} .
$$

Therefore

$$
\left\|\psi^{\prime}\right\|_{L^{q}} \leqslant \frac{1}{\mu-1}\left\|\psi^{\prime \prime}\right\|_{L^{q}}+\frac{2 \mu(\mu+1)}{\mu-1}\|\psi\|_{L^{q}} .
$$

For a fixed number $v_{0}>0$, put

$$
C=\max _{\left[1+v_{0}, \infty[\right.} \frac{\mu(\mu+1)}{(\mu-1)^{2}},
$$

then, for all $\mu \geqslant 1+v_{0}$

$$
\frac{2 \mu(\mu+1)}{\mu-1} \leqslant 2 C(\mu-1) \text {. }
$$

Thus

$$
\forall \omega>0, \quad\left\|\psi^{\prime}\right\|_{L^{q}} \leqslant \omega\left\|\psi^{\prime \prime}\right\|_{L^{q}}+\frac{2 C}{\omega}\|\psi\|_{L^{q}} .
$$

Now, for $\omega=\sqrt{\frac{2 C\|\|_{L q}}{\left\|\psi^{\prime \prime}\right\|_{L^{q}}}}$, we get

$$
\left\|\psi^{\prime}\right\|_{L^{q}} \leqslant 2 \sqrt{2} \sqrt{C}\left\|\psi^{\prime \prime}\right\|_{L^{q}}^{1 / 2}\|\psi\|_{L^{q}}^{1 / 2}
$$

Lemma 14. There exists a constant $K>0$, such that for all function $w \in D(A)$, one has

$$
\|w\|_{F} \leqslant K\|w\|_{E_{\mathrm{hol}}}^{1 / 2}\|A w\|_{E_{\mathrm{hol}}}^{1 / 2} .
$$

Proof. Recall that

$$
\|w\|_{F}=\|w\|_{h_{0}^{\sigma}\left(W_{0}^{1, q}(0,1)\right)}+\left\|t^{-2 \alpha} w\right\|_{h_{0}^{\sigma}\left(W_{0}^{1, q}(0,1)\right)}
$$

and

$$
\left\{\begin{array}{l}
D(A)=\left\{w \in E_{\mathrm{hol}}: \forall t \in[0,1] w(t) \in D(L)\right\} \\
(A w)(t)=L(w(t)), \quad t \in[0,1]
\end{array}\right.
$$

Let $w \in D(A)$. Then

$$
\|w\|_{h_{0}^{\sigma}\left(W_{0}^{1, q}(0,1)\right)}=\max _{t \in[0,1]}\|w(t)\|_{W_{0}^{1, q}(0,1)}+\sup _{t \neq s} \frac{\|w(t)-w(s)\|_{W_{0}^{1, q}(0,1)}}{\|t-s\|^{\sigma}} .
$$


By virtue of Lemma 13, we have

$$
\|w(t)\|_{W_{0}^{1, q}(0,1)} \leqslant K\|w(t)\|_{L^{q}}^{1 / 2}\left\|D_{y}^{2} w(t)\right\|_{L^{q}}^{1 / 2},
$$

from which, we deduce that

$$
\begin{aligned}
\max _{t \in[0,1]}\|w(t)\|_{W_{0}^{1, q}(0,1)} & \leqslant K\left(\max _{t \in[0,1]}\|w(t)\|_{L^{q}}\right)^{1 / 2}\left(\max _{t \in[0,1]}\left\|D_{y}^{2}(w(t))\right\|_{L^{q}}\right)^{1 / 2} \\
& \leqslant K\left(\max _{t \in[0,1]}\|w(t)\|_{L^{q}}\right)^{1 / 2}\left(\max _{t \in[0,1]}\|(A w)(t)\|_{L^{q}}\right)^{1 / 2} \\
& \leqslant K\|w\|_{h_{0}^{\sigma}\left(L^{q}\right)}^{1 / 2}\|A w\|_{h_{0}^{\sigma}\left(L^{q}\right)}^{1 / 2} \leqslant K\|w\|_{h_{0}^{\sigma}\left(C_{0,0}\right)}^{1 / 2}\|A w\|_{h_{0}^{\sigma}\left(C_{0,0}\right)}^{1 / 2}
\end{aligned}
$$

and

$$
\begin{aligned}
\frac{\|w(t)-w(s)\|_{W_{0}^{1, q}(0,1)}}{\|t-s\|^{\sigma}} \leqslant & K\left(\frac{\|w(t)-w(s)\|_{L^{q}}}{\|t-s\|^{\sigma}}\right)^{1 / 2}\left(\frac{\|(A w)(t)-(A w)(s)\|_{L^{q}}}{\|t-s\|^{\sigma}}\right)^{1 / 2} \\
\leqslant & K\left(\sup _{t \neq s} \frac{\|w(t)-w(s)\|_{L^{q}}}{\|t-s\|^{\sigma}}\right)^{1 / 2} \\
& \times\left(\sup _{t \neq s} \frac{\|(A w)(t)-(A w)(s)\|_{L^{q}}}{\|t-s\|^{\sigma}}\right)^{1 / 2} .
\end{aligned}
$$

Hence

$$
\begin{aligned}
{[w]_{h_{0}^{\sigma}\left(W_{0}^{1, q}\right)} \leqslant K[w]_{h_{0}^{\sigma}\left(L^{q}\right)}^{1 / 2}[A w]_{h_{0}^{\sigma}\left(L^{q}\right)}^{1 / 2} } & \leqslant K\|w\|_{h_{0}^{\sigma}\left(L^{q}\right)}^{1 / 2}\|A w\|_{h_{0}^{\sigma}\left(L^{q}\right)}^{1 / 2} \leqslant K\|w\|_{h_{0}^{\sigma}\left(C_{0,0}\right)}^{1 / 2}\|A w\|_{h_{0}^{\sigma}\left(C_{0,0}\right)}^{1 / 2} .
\end{aligned}
$$

Thus, we obtain a part of the convexity inequality

$$
\|w\|_{h_{0}^{\sigma}\left(W_{0}^{1, q}(0,1)\right)} \leqslant K\|w\|_{h_{0}^{\sigma}\left(C_{0,0}\right)}^{1 / 2}\|A w\|_{h_{0}^{\sigma}\left(C_{0,0}\right)}^{1 / 2} \leqslant K\|w\|_{E_{\mathrm{hol}}}^{1 / 2}\|A w\|_{E_{\mathrm{hol}}}^{1 / 2} .
$$

Using the same method for the term $\left\|t^{-2 \alpha} w\right\|_{h_{0}^{\sigma}\left(W_{0}^{1, q}(0,1)\right)}$, we can show that

$$
\begin{aligned}
\left\|t^{-2 \alpha} w\right\|_{h_{0}^{\sigma}\left(W_{0}^{1, q}(0,1)\right)} & \leqslant K\left\|t^{-2 \alpha} w\right\|_{h_{0}^{\sigma}\left(C_{0,0}\right)}^{1 / 2}\left\|t^{-2 \alpha} A w\right\|_{h_{0}^{\sigma}\left(C_{0,0}\right)}^{1 / 2} \\
& \leqslant K\left\|t^{-2 \alpha} w\right\|_{E_{\mathrm{hol}}}^{1 / 2}\left\|t^{-2 \alpha} A w\right\|_{E_{\mathrm{hol}}}^{1 / 2} .
\end{aligned}
$$

This completes the proof of the lemma.

Finally, Theorem 6 leads to

Proposition 15. For $h \in E$, Problem (6) admits a unique strong solution $w=w_{1}=w_{2}$ fulfilling the following regularity 


$$
\left\{\begin{array}{l}
w \in h_{0}^{\sigma}\left([0,1] ; W_{0}^{1, q}(0,1)\right) \\
t^{-2 \alpha} w \in h_{0}^{\sigma}\left([0,1] ; W_{0}^{1, q}(0,1)\right)
\end{array}\right.
$$

for all $q>1$.

\subsection{Other regularities}

In this paragraph we analyze more regularities for the strong solution $w$ by inverting the variables $t, y$. We write the problem

$$
\left\{\begin{array}{l}
t^{2 \alpha} D_{t} w(t, y)-D_{y}^{2} w(t, y)+\lambda w(t, y)=h(t, y), \\
w_{\mid \partial \Omega-\Gamma_{1}}=0
\end{array}\right.
$$

we have studied, in the form

$$
\left\{\begin{array}{l}
t^{2 \alpha} D_{t} W(y, t)-D_{y}^{2} W(y, t)+\lambda W(y, t)=H(y, t), \\
W_{\mid \partial \Omega-\gamma_{1}}=0
\end{array}\right.
$$

where

$$
W(y, t)=w(t, y), \quad H(y, t)=h(t, y)
$$

and $\left.\gamma_{1}=\right] 0,1[\times\{1\}$ in variables $(y, t)$. By the anisotropy Lemma 4 , we also have

$$
H \in h_{0}^{\sigma}(\bar{\Omega})=L^{\infty}\left(0,1 ; h_{0}^{\sigma}([0,1])\right) \cap h_{0,0}^{\sigma}\left([0,1] ; C_{0}([0,1])\right)
$$

with respect to the variables $(y, t)$. Now, Problem (15) will be regarded as a second order differential equation of elliptic type for which we can apply the optimal results obtained in [4]. Let $X=C_{0}([0,1])$, we then rewrite (15) in the space $X$ in the following abstract form

$$
\left\{\begin{array}{l}
D_{y}^{2} W(y)+Q(W(y))=-H(y) \\
W(0)=W(1)=0
\end{array}\right.
$$

where we denote as usual $W(y)=W(y,$.$) . Here H \in h_{0,0}^{\sigma}([0,1] ; X)$.

\subsubsection{First case}

Define in $X=C_{0}([0,1])$, the operator $Q$ by

$$
\left\{\begin{array}{l}
D(Q)=C^{1}([0,1]) \cap C_{0}([0,1]), \\
(Q \psi)(t)=-t^{2 \alpha} \psi^{\prime}(t)-\lambda \psi(t), \quad t \in[0,1],
\end{array}\right.
$$

where as above, we assume that $\lambda$ is a fixed number in $] 0,2 \alpha-1[$.

Now, for $x \in C_{0}([0,1])$, the spectral equation

$$
Q \psi-z \psi=x
$$


is equivalent to

$$
\left\{\begin{array}{l}
t^{2 \alpha} \psi^{\prime}(t)+\lambda \psi(t)+z \psi(t)=-x(t), \\
x(0)=0
\end{array}\right.
$$

Thus for any $z \geqslant 0$, we have

$$
\psi(t)=-\int_{0}^{t}\left(\frac{x(\tau)}{\tau^{2 \alpha}} \mathrm{e}^{-(z+\lambda) \int_{\tau}^{t} s^{-2 \alpha} \mathrm{d} s}\right) \mathrm{d} \tau
$$

and

$$
\begin{aligned}
\|\psi(t)\| & \leqslant \int_{0}^{t}\|x(\tau)\| \tau^{-2 \alpha} \mathrm{e}^{-(z+\lambda) \int_{\tau}^{t} s^{-2 \alpha} \mathrm{d} s} \mathrm{~d} \tau \\
& \leqslant\|x\|_{X} \int_{0}^{t} \tau^{-2 \alpha} \mathrm{e}^{-(z+\lambda) \int_{\tau}^{t} s^{-2 \alpha} \mathrm{d} s} \mathrm{~d} \tau \\
& \leqslant\|x\|_{X} \int_{0}^{t} \tau^{-2 \alpha} \mathrm{e}^{\frac{(z+\lambda)}{2 \alpha-1}\left(t^{1-2 \alpha}-\tau^{1-2 \alpha}\right)} \mathrm{d} \tau \\
& \leqslant\|x\|_{X} \mathrm{e}^{\frac{(z+\lambda)}{2 \alpha-1} t^{1-2 \alpha}} \int_{0}^{t} \tau^{-2 \alpha} \mathrm{e}^{\frac{-(z+\lambda)}{2 \alpha-1} \tau^{1-2 \alpha}} \mathrm{d} \tau \\
& \leqslant \frac{\|x\|_{X}}{(z+\lambda)},
\end{aligned}
$$

this implies that the operator $Q$ verifies

$$
\left\{\begin{array}{l}
\exists C>0: \forall z \geqslant 0 \quad(Q-z I)^{-1} \in L(X) \quad \text { and } \\
\left\|(Q-z I)^{-1}\right\|_{L(X)} \leqslant \frac{C}{1+z} .
\end{array}\right.
$$

In addition the two compatibility conditions

$$
\left\{\begin{array}{l}
-H(0)-Q(0)=0 \in \overline{D(Q)}=X, \\
-H(1)-Q(1)=0 \in \overline{D(Q)}=X
\end{array}\right.
$$

are satisfied. Hence the results in [4,5] apply and give

Proposition 16. For $H \in h_{0,0}^{\sigma}([0,1] ; X)$, there exists a unique strict solution

$$
W \in C^{2}([0,1] ; X) \cap C_{0,0}([0,1] ; D(Q))
$$

such that

(i) $D_{y}^{2} W, Q(W().) \in h^{\sigma}([0,1] ; X)$,

(ii) $\sup _{y \in[0,1]}\left\|D_{y}^{2} W(y)\right\|_{D_{Q}(\sigma)}<\infty$.

It is well known that the little real interpolation space $D_{Q}(\sigma)$ cited in this proposition (with respect to the variable $t$ ), coincides exactly with 


$$
D_{Q}(\sigma)=h_{0}^{\sigma}([0,1])
$$

(See Lunardi [8]).

Applying the regularities in statement (i) to $w$ with respect to the variables $(t, y)$ we deduce that

$$
\left\{\begin{array}{l}
D_{y}^{2} w \in C\left([0,1] ; h^{\sigma}([0,1]),\right. \\
w \in C^{1}\left([0,1] ; h^{\sigma}([0,1]) .\right.
\end{array}\right.
$$

The "double" regularity in statement (ii) means that

$$
D_{y}^{2} W \in L^{\infty}\left(0,1 ; h^{\sigma}([0,1])\right.
$$

(with respect to the variables $(y, t)$ ), from which, and after going back to $w$, we get

$$
D_{y}^{2} w \in h^{\sigma}([0,1] ; C([0,1])
$$

(with respect to the variables $(t, y)$ ).

\subsubsection{Second case}

In the same manner, Problem (16) can be considered in the space

$$
L^{\infty}(0,1 ; X)
$$

with the second member verifying

$$
H \in L^{\infty}\left(0,1 ; h_{0}^{\sigma}([0,1])\right)=L^{\infty}\left(0,1 ; D_{Q}(\sigma)\right),
$$

and also the results in [5] apply to give

Proposition 17. For $H \in L^{\infty}\left(0,1 ; D_{Q}(\sigma)\right)$, there exists a unique strict solution

$$
W \in W^{2, \infty}(0,1 ; X) \cap L^{\infty}(0,1 ; D(Q))
$$

such that $D_{y}^{2} W, Q W(.) \in L^{\infty}\left(0,1 ; h^{\sigma}([0,1])\right)$.

One deduces that (with respect to the variables $(t, y)$ )

$$
\left\{\begin{array}{l}
D_{y}^{2} w \in L^{\infty}\left(0,1 ; h_{0}^{\sigma}([0,1]),\right. \\
D_{t} w \in L^{\infty}\left(0,1 ; h_{0}^{\sigma}([0,1]) .\right.
\end{array}\right.
$$

\subsection{Back to $v$}

Summarizing all the results on $w$ obtained by the sum theory, we have

$$
\left\{\begin{array}{l}
\text { (i) } w \in L^{\infty}\left(0,1 ; h_{0,0}^{\sigma}([0,1])\right), \\
\text { (ii) } w \in h_{0}^{\sigma}\left([0,1] ; C_{0,0}([0,1])\right), \\
\text { (iii) } w \in h_{0}^{\sigma}\left([0,1] ; W_{0}^{1, q}(0,1)\right), \\
\text { (iv) } t^{-2 \alpha} w \in L^{\infty}\left(0,1 ; h_{0,0}^{\sigma}([0,1])\right), \\
\text { (v) } t^{-2 \alpha} w \in h_{0}^{\sigma}\left([0,1] ; W_{0}^{1, q}(0,1)\right) .
\end{array}\right.
$$


We recall that

$$
v(t, y)=\mathrm{e}^{\lambda \frac{t^{1-2 \alpha}}{(1-2 \alpha)}} w(t, y)
$$

where $\lambda$ is a fixed number in $] 0,2 \alpha-1[$.

Since the following functions

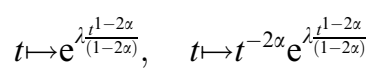

are in $C_{b}^{1}([0,1])$, it is not difficult to obtain the above same regularities on $v$. Then by virtue of Lemma 4 and the fact that $W_{0}^{1, q}(0,1)$ is continuously imbedded in $C_{0,0}([0,1])$, we deduce

$$
\left\{\begin{array}{l}
v \in h_{0}^{\sigma}(\bar{\Omega}), \\
t^{-2 \alpha} v \in h_{0}^{\sigma}(\bar{\Omega}), \\
v \in h_{0}^{\sigma}\left([0,1] ; W_{0}^{1, q}(0,1)\right), \\
t^{-2 \alpha} v \in h_{0}^{\sigma}\left([0,1] ; W_{0}^{1, q}(0,1)\right) .
\end{array}\right.
$$

Now using the regularities obtained by the approach given in [4,5], we have

$$
\left\{\begin{array}{l}
D_{y}^{2} v \in C\left([0,1] ; h^{\sigma}([0,1]),\right. \\
v \in C^{1}\left([0,1] ; h^{\sigma}([0,1]),\right. \\
D_{y}^{2} v \in h^{\sigma}([0,1] ; C([0,1]), \\
D_{y}^{2} v \in L^{\infty}\left(0,1 ; h^{\sigma}([0,1]),\right. \\
D_{t} v \in L^{\infty}\left(0,1 ; h^{\sigma}([0,1])\right.
\end{array}\right.
$$

from which we deduce by in virtue of Lemma 4 that

$$
\left\{\begin{array}{l}
D_{y}^{2} v \in h_{0}^{\sigma}(\bar{\Omega}), \\
t^{2 \alpha} D_{t} v \in h_{0}^{\sigma}(\bar{\Omega}) .
\end{array}\right.
$$

Summing up (18) and (19), we obtain Theorem 1.

\section{Remark 18}

1. Note that the first and second application of the sum theory was useful to obtain the vector-valued weight regularities (with respect to $t^{-2 \alpha}$ ).

2. The previous study can be extended in a natural way to the degenerate parabolic problems of general form

$$
\left\{\begin{array}{l}
\varphi(t)^{2} D_{t} v(t)+L v(t)=k(t) \\
v(0)=0
\end{array}\right.
$$

where $L$ is an operator of general elliptic type with the fundamental assumption: $\varphi \varphi^{\prime}$ is a Hölder continuous function. 


\section{References}

[1] T. Berroug, Sur des problèmes elliptiques et paraboliques dans les espaces de Hölder et les petits Hölder, Thèse de Doctorat, Université du Havre, 2003.

[2] G. Da Prato, P. Grisvard, Sommes d'opé rateurs linéaires et equations différentielles opérationnelles, J. Math. Pure Appl. IX 54 (1975) 305-387.

[3] A. Favini, A. Yagi, Multivalued linear operators and degenerate evolution equations, Ann. Mat. Pura Appl. (IV) 163 (1993) 353-384.

[4] R. Labbas, Equation elliptique abstraite du second ordre et equation parabolique pour le problème de Cauchy abstrait, C.R. Acad. Sci. Paris 305 (I) (1987) 785-788.

[5] A. El Haial, R. Labbas, On the ellipticity and solvability of abstract second-order differential equation, Electron. J. Diff. Eqs. 57 (2001) 1-18.

[6] R. Labbas, Some results on the sum of linear operator with nondense domains, Ann. Mat. Pura Appl. IV CLIV (1989) 91-97.

[7] R. Labbas, A. Medeghri, B.-K. Sadallah, Sur une equation parabolique dans un domaine non cylindrique, C.R. Acad. Sci. Paris 335 (I) (2002) 1017-1022.

[8] A. Lunardi, Analytic Semi-Groups and Optimal Regularity in Parabolic Problems, Birkäuser, Boston, 1995.

[9] M. Najmi, Régularité-Singularité dans les espaces de Hölder pour un problème elliptique et l'equation de la chaleur dans des domaines non réguliers, Thèse d'état, Université de Nice, 1992. 